\title{
Advances in epithelial ovarian cancer
}

Deborah Neesham, Anthony Richards, Melissa McGauran

\section{Background}

Most epithelial ovarian cancer occurs in older women, with a mean age at diagnosis of 62 years and an overall five-year survival rate in Australia of $43 \%$. Most women are diagnosed with advanced disease of high-grade serous type with $20-30 \%$ five-year survival; $70 \%$ relapse within three years of initial treatment. There is no available screening test for ovarian cancer.

\section{Objective}

The aim of this article is to highlight current management and future directions for women diagnosed with epithelial ovarian cancer, particularly the high incidence of underlying genetic mutations and new options for treatment.

\section{Discussion}

Risk-reducing surgery with bilateral salpingo-oophorectomy is recommended for women at high risk of developing ovarian cancer. Ovarian cancer treatment still centres on surgery and chemotherapy, with aggressive cytoreductive techniques and intraperitoneal treatments being evaluated in advanced disease. Molecular targeting agents are revolutionising treatment options, particularly the poly adenosine diphosphate-ribose polymerase inhibitors, and especially for patients with an underlying $B R C A$ mutation. Other molecular targeting agents, such as vascular endothelial growth factor (VEGF) receptor inhibitors and newer approaches using immunotherapy and molecular targeting, aim to individualise treatment and improve survival in the future.
MOST OVARIAN CANCER Occurs in older women, with a mean age at diagnosis of 62 years and an overall five-year survival rate in Australia of $43 \% .^{1}$ Most women are diagnosed with advanced disease of high-grade serous (HGS) type with 20-30\% five-year survival, as $70 \%$ relapse within three years of initial treatment. ${ }^{2}$ There is no available screening for ovarian cancer, and risk-reducing surgery with bilateral salpingo-oophorectomy is the best option for women at high risk. ${ }^{3}$ Ovarian cancer treatment still centres on surgery and chemotherapy, with aggressive cytoreductive techniques and intraperitoneal treatments being evaluated in advanced disease. Molecular targeting agents are revolutionising treatment options, particularly poly adenosine diphosphate-ribose polymerase (PARP) inhibitors (PARPis), especially for patients with an underlying $B R C A$ mutation. Other molecular targeting agents - such as vascular endothelial growth factor (VEGF) receptor inhibitors and newer approaches with immunotherapy and molecular targeting - aim to individualise treatment and improve survival in the future.

\section{Screening, prevention and detection}

\section{Screening}

There is no proven effective screening test available to diagnose premalignant or early-stage disease in the general population or in women at high risk. ${ }^{3}$
Given the current theory that most HGS ovarian cancers are derived from the fimbrial end of the fallopian tube (Figure 1), it is unlikely that a premalignant phase will release proteins or DNA signatures into the bloodstream for early detection. ${ }^{4}$

\section{Potential prevention strategies}

Potential prevention strategies include:

- oral contraceptive pill - associated with a $10 \%$ reduction in risk of ovarian cancer per year of use, up to a risk reduction of $60-70 \%{ }^{5}$

- tubal ligation or hysterectomy associated with up to a $30 \%$ reduction in risk of developing ovarian cancer ${ }^{6,7}$

- salpingectomy - it has been suggested that any woman having a hysterectomy should be counselled about having her fallopian tubes removed during the same surgery ${ }^{8}$ while conserving normal ovaries, which may have a function into the patient's mid-60s in terms of cardiovascular health and prevention of osteoporosis. ${ }^{9}$

\section{Detection}

The primary imaging investigation should be pelvic ultrasonography to assess the characteristics of a mass (assessing solid areas, complexity and increased blood flow) and look for ascites. If there is a suspicion of malignancy, further imaging of the chest, abdomen and pelvis is usually performed with computed tomography (CT). Magnetic resonance imaging (MRI) is helpful in assessing pelvic masses but does not attract a 
rebate through the Medicare Benefits Schedule (MBS). A positron emission tomography (PET) scan is not indicated for diagnosis, although it may be used to assess metastatic disease following diagnosis. However, it is difficult to detect miliary peritoneal disease with all imaging modalities including PET.

The cancer antigen 125 (CA125) tumour marker is not useful for screening or early detection as it is not elevated in $50 \%$ of women with stage 1 ovarian cancer, although it is raised in $>90 \%$ of women with advanced stage disease. ${ }^{3}$ CA125 may be elevated in many other situations apart from ovarian cancer, particularly during pre-menopause with endometriosis and other benign cysts. ${ }^{10}$ It is, however, useful as a baseline measure for monitoring response to treatment.

The carcinoembryonic antigen may be elevated in women with mucinous tumours, but these account for only $3 \%$ of primary ovarian cancers. ${ }^{11}$

If there is an acute presentation in a woman aged $<35$ years, it is important to test for the relevant germ cell tumour markers, human chorionic gonadotrophin, alpha-fetoprotein and lactate dehydrogenase.

\section{Genetic testing}

BRCA1, BRCA2 and Lynch syndrome (mismatch repair protein loss) are three known autosomal dominant germline mutations imparting a high risk of ovarian cancer, with lifetime risks of developing ovarian cancer of $44 \%, 17 \%$ and $12 \%$ respectively. ${ }^{12,13}$ This can be compared with the general population risk of $1.4 \%$, and the risk for a woman with a first-degree relative of 3-4\%. ${ }^{14}$

Women who potentially have a high risk of ovarian cancer include those with:

- a first-degree relative aged $<60$ years at diagnosis

- more than one first- or second-degree relative on the same side of the family diagnosed at any age with invasive epithelial ovarian cancer

- a strong family history of both breast and ovarian cancer

- a family history of breast and/or ovarian cancer with Ashkenazi Jewish heritage.

These women should be referred for genetic counselling. ${ }^{3}$

Women of Ashkenazi Jewish descent have a one in 40 risk of carrying a $B R C A$ mutation (compared with the general population risk of one in 500). ${ }^{15} \mathrm{~A}$ current pilot project in Sydney and Melbourne is assessing the acceptability and feasibility of looking for founder mutations on buccal swabs in these communities (www.jenescreen.com.au).

There is also an Australian Government-funded initiative called TRACEBACK that aims to reduce the number of new ovarian and breast cancers diagnosed in Australia by identifying families that may have a hereditary risk of cancer development due to inherited mutations. TRACEBACK offers testing for women who have previously been diagnosed with ovarian cancer but have not been tested for mutations. TRACEBACK also assists people with a family member who died from ovarian cancer without having genetic testing to try to access archived tissue samples for testing. Participation can be arranged by contacting Ovarian Cancer Australia.

All women diagnosed with nonmucinous grade 2-3 epithelial ovarian cancer are eligible for MBS-funded mainstream genetic testing, as the risk of a germline mutation in these women is $15 \% .{ }^{16}$ Mainstream testing is the integration of genetic testing within the treating oncology team, without prior referral to a genetic counsellor. Results available by the end of primary treatment may inform maintenance treatment as

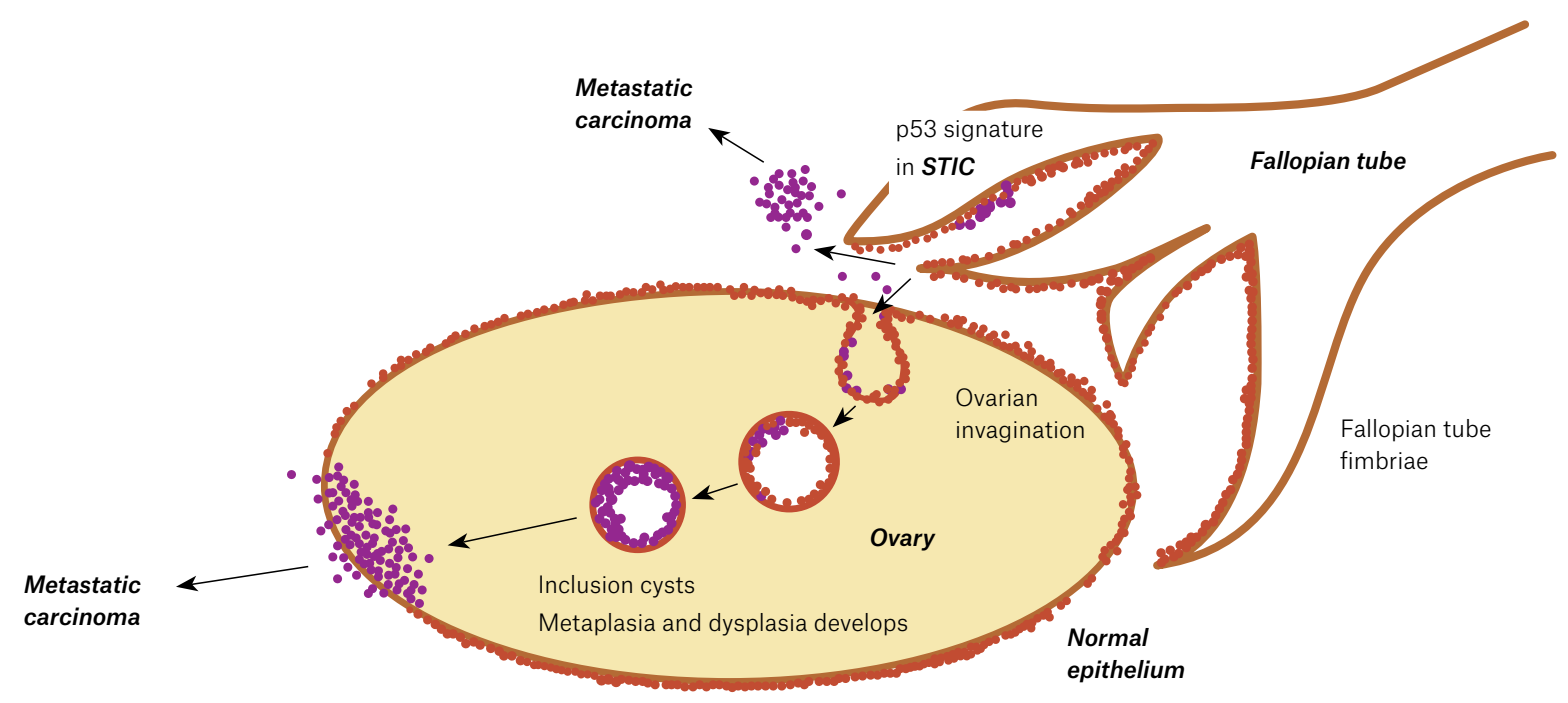

Figure 1. Aetiology of high-grade serous ovarian cancer STIC, serous tubal intraepithelial carcinoma 
well as providing timely access to other family members for counselling and genetic testing.

\section{Early-stage disease surgical management}

Epithelial ovarian cancer is uncommonly diagnosed at an early stage. On full surgical staging of apparently early-stage tumours, $20-30 \%$ will be upstaged. ${ }^{17}$ The less common epithelial subtypes (ie clear cell, endometrioid and mucinous tumours) are more likely to be diagnosed at an early stage.

The option for fertility sparing with conservation of the uterus and the other macroscopically normal ovary may be available in early-stage disease, although women should be counselled that endometrioid and serous tumours have a $10-20 \%$ risk of bilaterality. Chemotherapy, if required, does not preclude fertility.

\section{Late-stage disease surgical management}

Management of advanced ovarian cancer combines chemotherapy with surgery. Many studies have shown that the most important prognostic indicator for survival in advanced ovarian cancer is the amount of residual disease at the end of cytoreductive surgery. ${ }^{18}$ There is limited evidence to support the conclusion that the surgical procedure is directly responsible for the superior outcome associated with less residual disease; however, if there is no macroscopic residual disease, the median five-year survival is $60 \%{ }^{19}$

If on initial imaging there is an expectation of no residual disease and the patient is suitable for surgery, then cytoreductive surgery is recommended. This pre-operative imaging assessment is often difficult because small volume miliary disease is often undetectable on CT, MRI or PET scanning. A diagnostic laparoscopy may be used to assess suitability for upfront surgery. Some factors definitively determine the inability to remove the entire tumour, such as involvement of the porta hepatis, small bowel mesentery or small bowel serosa, or stage 4 disease with pleural involvement.
If, based on imaging, there is no chance of removing all macroscopic disease at the initial surgery, then neoadjuvant chemotherapy has been shown in prospective randomised trials to have equivalent survival with reduced morbidity. The surgery required is less radical and the patient is in a better physiological state, with reduced tumour burden and ascites after three cycles of chemotherapy. ${ }^{20}$ Accordingly, where there is an expectation of significant unresectable disease, there is a trend to neoadjuvant chemotherapy with three cycles of chemotherapy then interval debulking surgery followed by another three cycles of chemotherapy post-surgery.

If there is chemotherapy-resistant or refractory disease, the role of surgery is unclear, as it may offer no survival benefit. In the USA, up to $20 \%$ of women do not have surgery at any point. ${ }^{21}$

\section{Adjuvant treatment}

Current standard adjuvant therapy for advanced ovarian cancer is six cycles of chemotherapy with carboplatin and paclitaxel given three times weekly. ${ }^{22}$

Maintenance bevacizumab (a VEGF receptor inhibitor) has been shown to prolong disease-free survival (DFS) by four months in women with stage 4 disease or suboptimally ( $>1 \mathrm{~cm}$ residual) debulked stage $3 \mathrm{C}$ disease when given intravenously (IV) every three weeks with chemotherapy and for up to 12 months thereafter. It improved overall survival by 10 months in stage 4 disease (from 33 to 43 months) but had no effect on overall survival in stage $3 \mathrm{C}$ disease. ${ }^{23}$

Individualisation of treatment with targeted therapies depends on detecting molecular targets within tumour tissue. At a molecular level, homologous recombination deficiencies (HRDs) are seen in approximately $50 \%$ of ovarian cancers, primarily through $B R C A$ germline (gBRCA) mutations (20\%). Nine per cent of ovarian cancers have a somatic (non-inherited, tumour-specific) BRCA mutation, and $18 \%$ have other HRD mutations, which makes both groups respond to molecular targeted therapies that inhibit the enzyme PARP. ${ }^{24}$
Accordingly, PARPis are particularly active in $\mathrm{gBRCA}$ mutation carriers and also show activity in women with somatic BRCA (sBRCA) mutations and HRDs, but at a lower rate. Olaparib is one PARPi that, when used as oral maintenance treatment in $\mathrm{g} B R C A$ mutation carriers, has shown $60 \%$ DFS at three years, compared with $27 \%$ in controls. ${ }^{25}$ Other PARPis investigated include niraparib and rucaparib. In trials of maintenance rucaparib after response to two lines of platinum chemotherapy, DFS was improved in BRCA mutation carriers (16.6 months) when compared with patients with HRDs (13.6 months) and controls (5.4 months). ${ }^{26}$ The main side effects of PARPis are fatigue, gastrointestinal upset and anaemia. PARPis are the most outstanding advance in ovarian cancer control in recent years.

Other novel therapies currently in clinical trials include immunotherapies with monoclonal antibodies such as nivolumab and ipilumumab; however, there are currently no randomised controlled trials (RCTs) showing a progression-free survival (PFS) or overall survival benefit from these.

\section{Intraperitoneal chemotherapy and HIPEC}

Intraperitoneal chemotherapy has been used in ovarian cancer for many years, with part of each treatment cycle given through a port implanted at the time of surgery. Studies have shown an outcome benefit, but barriers to widespread uptake include increased toxicity, inconvenience and extra cost. ${ }^{27}$

Intraperitoneal chemotherapy can also be administered as part of maximal cytoreductive surgery at the time of peritonectomy using heated intraperitoneal chemotherapy (HIPEC). This requires significant resources including access to an intensive care unit. A recent Dutch RCT of 245 patients undergoing interval cytoreductive surgery showed an improvement in PFS and five-year survival in women treated with HIPEC at the time of surgery (PFS 14.2 months, compared with 10.7 months in patients not treated with HIPEC, and survival at five years of 
$50 \%$, compared with $38 \%$ in patients not treated with HIPEC). ${ }^{28}$ There are many RCTs underway, and there is interest in peritonectomy and HIPEC in Australia; however, these treatments should only be offered in a clinical trial setting. ${ }^{29}$

\section{Treatment of recurrent disease}

Recurrence will occur in approximately $70 \%$ of women diagnosed with epithelial ovarian cancer, ${ }^{30}$ at which time quality of life becomes the main goal of treatment.

Most recurrences are diagnosed with an asymptomatic rise in CA125 levels three months prior to symptom development. If there is no potential for resection, with commonly widespread intraperitoneal recurrence, systemic treatment will be required. There is no survival advantage in restarting systemic treatment before the development of symptoms. ${ }^{31}$

Hormonal treatment occasionally provides clinical benefit with acceptable toxicity. ${ }^{32}$

\section{Palliative treatments}

Chemotherapy is occasionally used for palliation of symptoms.

Ascitic taps may be required for comfort, and implantable drains may be required to avoid multiple paracenteses.

There are rare situations in which targeted radiotherapy for symptom control is used if solid masses are causing compressive or obstructive symptoms.

Early referral to palliative care services provides the best supportive care for women. However, as a result of a typically prolonged disease course with multiple lines of treatment, these women require a team approach, as for any patient with a chronic disease.

\section{Key points}

- Epithelial ovarian cancer is usually at an advanced stage at diagnosis, and the average patient age at diagnosis is 62 years. Epithelial ovarian cancer is uncommon (one in 85 lifetime risk for Australian women), but only $43 \%$ of patients survive five years. All patients should be managed in consultation with a gynaecological oncology unit and have their cases discussed at a multidisciplinary tumour board meeting.

- Women with persistent bloating, abdominal pain and bowel and bladder dysfunction present a diagnostic dilemma for clinicians.

- There is currently no screening or early diagnostic test available, even for women with a high risk of ovarian cancer.

- Bilateral salpingo-oophorectomy provides a $95 \%$ reduction in risk (residual 5\% due to primary peritoneal tumour development) for those with $B R C A$ or Lynch syndrome mutations. Opportunistic salpingectomy should be discussed with all women at the time of benign hysterectomy, given that the origin of most HGS tumours is thought to be the fallopian tube.

- BRCA mutations are common (15-20\%) in HGS cancers, and all women with primary nonmucinous epithelial ovarian cancer should be offered referral to a genetic counselling clinic. There is a dramatic improvement in DFS with PARPis for these women.

- Future advances in molecular profiling with potential for immunotherapy and molecular targeting therapy are promising for individualisation of treatment options.

- A team approach is important when managing women with epithelial ovarian cancer.

\section{Authors}

Deborah Neesham MBBS (Melb), DCH (London), FRANZCOG, CGO, Consultant Gynaecological Oncologist, The Women's Hospital, Frances Perry House, Vic. Neeshamd@ramsayhealth.com.au Anthony Richards MBBS, FRANZCOG, CGO, Consultant Gynaecological Oncologist, The Women's Hospital, Vic; Joan Kirner Women and Children's Hospital, Vic

Melissa McGauran MBBS, Registrar, Sunshine Hospital, Vic

Competing interests: None.

Funding: None.

Provenance and peer review: Commissioned, externally peer reviewed.

\section{References}

1. Arnold M, Rutherford MJ, Bardot A, et al. Progress in cancer survival, mortality and incidence in seven high-income countries 1995-2014 (ICBP
Survmark-2): A population based study. Lancet Oncol 2019;20(11):1493-505. doi: 10.1016/S14702045(19)30456-5.

2. Anuradha S, Webb PM, Blomfield P, et al. Survival of Australian women with invasive epithelial ovarian cancer: A population based study. Med J Aust 2014;201(5):283-88. doi: 10.5694/ mja14.00132.

3. Cancer Australia. Position Statement - Testing for ovarian cancer in asymptomatic women. Surry Hills, NSW: Cancer Australia, 2019.

4. Salvador S, Gilks B, Köbel M, Huntsman D, Rosen B, Miller D. The fallopian tube: Primary site of most pelvic high grade serous carcinomas. Int J Gynecol Cancer 2009;19(1):58-64. doi: 10.1111/ IGC.0b013e318199009c.

5. Havrilesky LJ, Moorman PJ, Lowery WJ, et al. Oral contraceptive pills as primary prevention for ovarian cancer: A systematic review and metaanalysis. Obstet Gynecol 2013;122(1):139-47. doi: 10.1097/AOG.0b013e318291c235.

6. Green A, Purdie D, Bain C, et al. Tubal sterilisation, hysterectomy and decreased risk of ovarian cancer. Survey of Women's Health Study Group. Int J Cancer 1997;71(6):948-51. doi: 10.1002/ (sici)1097-0215(19970611)71:6<948::aidijc6>3.0.co;2-y.

7. Cibula D, Widschwendter M, Majek O, Dusek L. Tubal ligation and the risk of ovarian cancer Review and meta analysis. Hum Reprod Update 2011;17(1):55-67. doi: 10.1093/humupd/dmq030.

8. Womens Health Committee. Managing the adnexae at the time of hysterectomy for benign gynaecological disease. East Melbourne, Vic: The Royal Australian and New Zealand College of Obstetricians and Gynaecologists, 2017.

9. Jacoby VL, Grady D, Wactawski-Wende J, et al. Oophorectomy vs ovarian conservation with hysterectomy: Cardiovascular disease, hip fracture, and cancer in the Women's Health Initiative Observational Study. Arch Intern Med 2011;171(8):760-68. doi: 10.1001/ archinternmed.2011.121

10. Moss EL, Hollingsworth J, Reynolds TM. The role of CA125 in clinical practice. J Clin Pathol 2005;58(3):308-12. doi: 10.1136/jcp.2004.018077.

11. Morice P, Gouy S, Leary A. Mucinous ovarian carcinoma. N Engl J Med 2019;380(13):1256-66. doi: 10.1056/NEJMra1813254.

12. Kuchenbaecker KB, Hopper JL, Barnes DR, et al. Risks of breast, ovarian and contralateral breast cancer for BRCA1 and BRCA2 mutation carriers. JAMA 2017;317(23):2402-16. doi: 10.1001/ jama.2017.7112.

13. Lynch HT, Casey MJ, Snyder CL, et al. Hereditary ovarian cancer: Heterogeneity, molecular genetics, pathology, and management. Mol Oncol 2009;3(2):97-137. doi: 10.1016/j. molonc.2009.02.004.

14. Soegard M, Frederiksen $K$, Jensen $A$, et al. Risk of ovarian cancer in first-degree relatives with cancer. Acta Obstetricia et Gynaecologica Scandinavica 2009;88(4):449-56. doi: 10.1080/00016340902807207.

15. Roa BB, Boyd AA, Volcik K, Richards CS. Ashkenazi Jewish population frequencies for common mutations in BRCA1 and BRCA2. Nat Genet 1996;14(2):185-87. doi: 10.1038/ng1096-185

16. Manchana T, Phoolcharoen N, Tantbirojn P. BRCA mutation in high grade epithelial ovarian cancers. Gynecol Oncol Rep 2019;29:102-05. doi: 10.1016/j. gore.2019.07.007.

17. Hengeveld EM, Zusterzeel PLM, Lajer $\mathrm{H}$, Hogdall CK, Rosendahl M. The value of surgical 
staging in patients with apparent early stage epithelial ovarian cancer. Gynecol Oncol 2019;154(2):308-13.

18. Du Bois A, Reuss A, Pujade-Lauraine E, Harter P, Ray-Coquard I, Pfisterer J. Role of surgical outcome as prognostic factor in advanced epithelial ovarian cancer: A combined exploratory analysis of 3 prospectively randomized phase 3 multicenter trials: By the Arbeitsgemeinschaft Gynaekologische Onkologie Studiengruppe Ovarialkarzinom (AGO-OVAR) and the Groupe d'Investigateurs Nationaux Pour les Etudes des Cancers de l'Ovaire (GINECO). Cancer 2009;115(6):1234-44. doi: 10.1002/cncr.24149.

19. Elattar A, Bryant A, Winter-Roach BA, Hatem M, Naik R. Optimal primary surgical treatment for advanced epithelial ovarian cancer. Cochrane Database Syst Rev 2011;(8):CD007565. doi: 10.1002/14651858.CD007565.pub2.

20. Vergote I, Tropé CG, Amant F, et al. Neoadjuvant chemotherapy or primary surgery in stage IIIC or IV ovarian cancer. N Engl J Med 2010;363(10):943-53. doi: 10.1056/NEJMoa0908806.

21. Shalowitz D, Epstein A, Ko E, Giuntoli R. Non-surgical management of ovarian cancer: Prevalence and implications. Gynecol Oncol 2016;142:30-37. doi: 10.1016/ygyno.2016.04.026.

22. Cancer Australia. First line chemotherapy for the treatment of women with epithelial ovarian cancer: Recommendations for the use of first-line chemotherapy for the treatment of women with epithelial ovarian cancer. Surry Hills, NSW: Cancer Australia, 2014.
23. Liu JF, Matulonis UA. Bevacizumab in newly diagnosed ovarian cancer. Lancet Oncol 2015;16(8);876-78. doi: 10.1016/S14702045(15)00064-9.

24. da Cunha Colombo Bonadio RR, Fogace RN, Miranda VC, Diz MDPE. Homologous recombination deficiency in ovarian cancer: A review of its epidemiology and management Clinics (Sao Paolo) 2018:73(Suppl 1):e450s. doi: 10.6061/clinics/2018/e450s.

25. Moore K, Colombo N, Scambia G, et al. Maintenance olaparib in patients with newly diagnosed advanced ovarian cancer. N Engl J Med 2018;379(26):2495-505. doi: 10.1056/ NEJMoa1810858.

26. Coleman RL, Oza AM, Lorusso D, et al. Rucaparib maintenance treatment for recurrent ovarian cancer after response to platinum therapy (Ariel3): A randomised, double-blind, placebo-controlled, phase 3 trial. Lancet 2017;390(10106):1949-91. doi: 10.1016/S0140-6736(17)32440-6.

27. Gourley C, Walker JL, Mackay HJ. Update on intraperitoneal chemotherapy for the treatment of epithelial ovarian cancer. Am Soc Clin Oncol Educ Book 2016;35:143-51. doi: 10.14694/ EDBK_158927.

28. van Driel WJ, Koole SN, Sikorska K, et al. Hyperthermic intraperitoneal chemotherapy in ovarian cancer. N Eng J Med 2018;378(3):230-40. doi: 10.1056/NEJMoa1708618.

29. Farrell R. Is peritonectomy and hyperthermic intraperitoneal chemotherapy a new standard of treatment for advanced epithelial ovarian cancer?
Aust N Z J Obstet Gynaecol 2019;59(3):335-40. doi: 10.1111/ajo.12953.

30. Ozols RF. Treatment goals in ovarian cancer Int J Gynaecol Cancer 2005;15 Suppl 1:3-11. doi: 10.1111/j.1525-1438.2005.15351.x.

31. Rustin GJ, van der Burg ME, Griffin CL, et al. Early versus delayed treatment of relapsed ovarian cancer (MRC OV05/ EORTC 55955): A randomised trial. Lancet 2010;376(9747):1155-63. doi: 10.1016/S01406736(10)61268-8.

32. Bonaventura A, O'Connell RL, Mapagu C, et al. Paragon(ANZGOG-0903): Phase 2 study of anastrozole in women with estrogen or progesterone receptor-positive platinumresistant or -refractory recurrent ovarian cancer. Int J Gynecol Cancer 2017;27(5):900-06. doi: 10.1097/IGC.0000000000000978. 